\title{
Trombosis séptica de senos craneales transverso y sigmoideo asociada a compromiso órbito-ocular en paciente pediátrico. Reporte de caso y revisión bibliográfica
}

\section{Septic thrombosis of the transverse and sigmoid cranial sinuses associated with ocular-orbital compromise in a pediatric patient. Case report and literature review}

\author{
Franco Benvenuto ${ }^{1 *}$, Mariana Sgroi, Soledad Guillénn ${ }^{1}$, Mauricio Galván-Chávez² y Adriana Fandiño \\ ${ }^{1}$ Servicio de Oftalmología, SAMIC Hospital Pediátrico Prof. Dr. JP Garrahan, Buenos Aires, Argentina; ${ }^{2}$ Departmento de Retina, Hospital Dr. Luis \\ Sánchez Bulnes de la Asociación Para Evitar la Ceguera en México I.A.P., Ciudad de México, México
}

\begin{abstract}
Resumen
Varón de 5 años con proptosis de 10 días de evolución, agudeza visual de no proyección luminosa y limitación a la abducción en el ojo derecho. El ojo izquierdo no presentaba alteraciones. En la ecografía ocular del ojo derecho se encontró un desprendimiento total de retina en embudo cerrado. En la resonancia magnética se observó un compromiso inflamatorio difuso de partes blandas en las órbitas. Se inició tratamiento antibiótico intravenoso con vancomicina y ceftazidima, con buena evolución clínica. Así mismo, la angiorresonancia registró un defecto del llenado central del seno sigmoideo y transverso derechos, compatible con trombosis. Se interpreta el cuadro como endoftalmitis y celulitis orbitaria de origen traumático con trombosis de senos transversos y sigmoideos derechos sin compromiso del seno cavernoso. La trombosis de senos transversos y sigmoideos es infrecuente, pero puede ser mortal. Actualmente, la resonancia magnética y la angiorresonancia han mejorado la capacidad de análisis de los senos venosos intracraneales.
\end{abstract}

Palabras clave: Proptosis. Celulitis. Trombosis venosa. Trauma ocular. Oftalmología pediátrica.

\begin{abstract}
This is the case of a 5-year-old male with a 10-day history of proptosis, with a visual acuity of no light perception and limited abduction in the right eye. The left eye was normal. The ocular ultrasound of the right eye revealed a total retinal detachment in a closed funnel. In Magnetic resonance imaging showed diffuse inflammatory involvement of the orbital tissues. Intravenous antibiotic treatment with vancomycin and ceftazidime was started, with good clinical evolution. Likewise, magnetic resonance angiography showed a central filling defect of the right sigmoid and transverse sinus, compatible with thrombosis. The diagnosis was an endophthalmitis and orbital cellulitis of traumatic origin with thrombosis of the transverse and right sigmoid sinuses without compromising the cavernous sinus. Transverse and sigmoid sinus thrombosis is rare but can be fatal. Currently, magnetic resonance imaging and resonance angiography have improved the ability to analyze the intracranial venous sinuses.
\end{abstract}

Keywords: Proptosis. Cellulitis. Venous thrombosis. Eye trauma. Pediatric ophthalmology.

Correspondencia:

${ }^{*}$ Franco Benvenuto

Pichincha 1850

Fecha de recepción: 19-09-2020

Disponible en internet: $25-10-2021$

C.P. 1245, Buenos Aires, Argentina

Fecha de aceptación: 06-04-2021

Rev Mex Oftalmol. (ahead of print)

DOI: 10.24875/RMO.M21000193

www.rmo.com.mx

0187-4519/๑ 2021 Sociedad Mexicana de Oftalmología. Publicado por Permanyer. Este es un artículo open access bajo la licencia CC BY-NC-ND (http://creativecommons.org/licenses/by-nc-nd/4.0/). 


\section{Introducción}

A pesar de que la incidencia de trombosis séptica de los senos venosos craneales ha disminuido drásticamente con el uso de antibióticos, la tasa de mortalidad sigue siendo alta. Las series más recientes estiman una tasa de mortalidad cercana al 19\% $\%^{1-3}$. Teichgraeber, et al. ${ }^{4}$ reportaron una tasa de mortalidad del $18-36 \%$. Las principales razones asociadas se deben al retraso en el diagnóstico por la falta de conocimiento de esta enfermedad y la forma de presentación clínica atípica, con síntomas y signos escasos debido a la terapia antibiótica indicada para controlar el foco de origen infeccioso ${ }^{1,2}$.

Los síntomas y signos mayormente reportados en la trombosis del seno transversal son dolor de cabeza, fiebre, secreción auditiva y dolor de oídos, y hallazgos ocasionales menos comunes que incluyen papiledema (20\% de los pacientes), vómitos, paresia del abducens y vértigo. El aumento de la presión intracraneal se produce en el $20-60 \%$ de los pacientes ${ }^{2,5}$.

El punto de partida de la infección por lo general es el macizo facial, y de forma más frecuente como complicación de una otomastoiditis. Las celulitis aisladas o junto con endoftalmitis rara vez producen entre sus complicaciones trombosis sépticas de senos venosos craneales, y si lo hacen, el primero en verse afectado por continuidad es el seno cavernoso $0^{6}$.

Se presenta el caso de un paciente pediátrico que desarrolló una trombosis séptica de senos transverso y sigmoideo como complicación de una celulitis asociada a una endoftalmitis por una herida órbito-ocular.

\section{Presentación del caso}

Varón de 5 años sin antecedentes personales ni familiares de interés que ingresa al servicio de oftalmología de nuestro hospital por derivación desde un centro de menor complejidad, para ser evaluado por sospecha de proceso oncológico ocular/orbitario por proptosis de 10 días de evolución. Se registra una agudeza visual de no proyección luminosa en el ojo derecho (OD) y 10/10 en el ojo izquierdo (OI), con cartilla de Snellen. El paciente tenía dificultad en las ducciones del $\mathrm{OD}$, principalmente limitación a la abducción. En el examen con lámpara de hendidura se observaron edema bipalpebral derecho, inyección conjuntival, quemosis, edema corneal, cámara anterior formada y catarata (Fig. 1). El fondo de ojo del OD no era evaluable. La biomicroscopía y el fondo de ojo del OI no presentaban alteraciones.

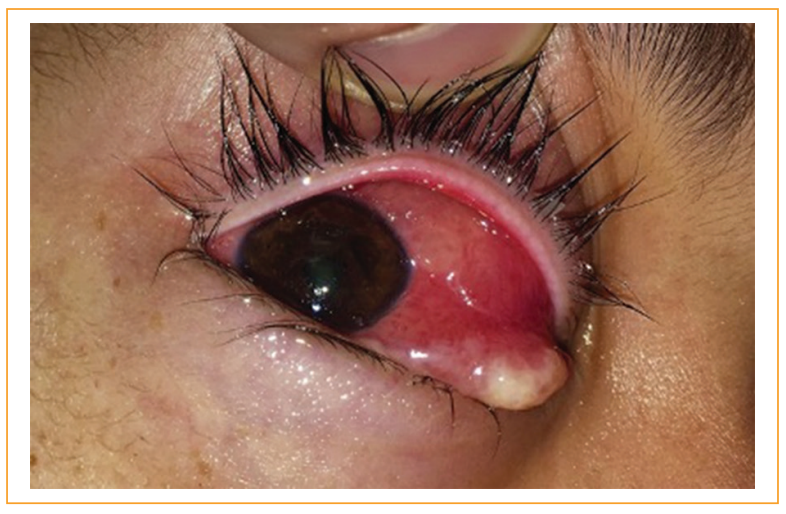

Figura 1. Foto clínica del ojo derecho, en el que se observan proptosis, edema bipalpebral, inyección conjuntival, quemosis, edema corneal, cámara anterior formada y catarata.

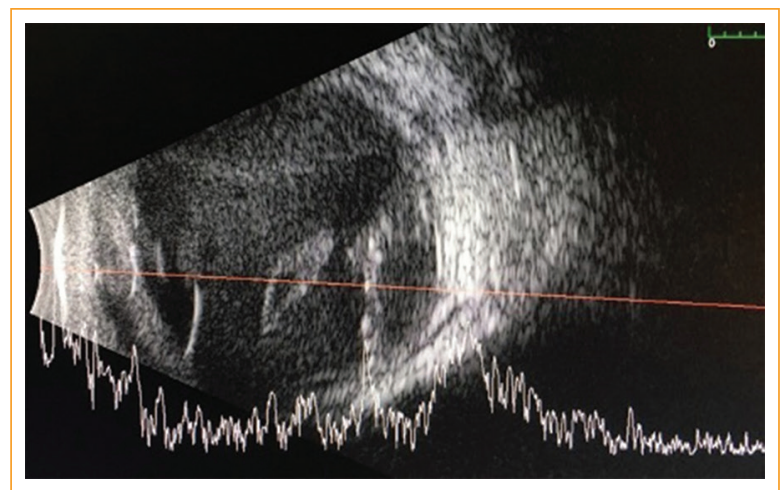

Figura 2. Ecografía del ojo derecho que muestra imágenes de tipo membrana de reflectividad mediaalta con posmovimientos rígidos, compatible con desprendimiento total de retina en embudo cerrado.

Se indica la internación del paciente, con evaluación clínica, estudios de laboratorios y resonancia magnética de encéfalo y órbita con protocolo oncológico. Como diagnósticos diferenciales de proptosis se plantearon tumores orbitarios, histiocitosis, retinoblastoma con invasión local, quistes, metástasis, celulitis y enfermedades oncohematológicas.

En el laboratorio se registraron leucocitosis y reactantes de fase aguda elevados. En la ecografía ocular del OD se observó desprendimiento total de retina en embudo cerrado (Fig. 2). En la resonancia magnética se objetivó compromiso inflamatorio difuso de partes blandas en las órbitas y un estafiloma anterior medial asociado a la inserción del recto medio, probablemente una solución de continuidad escleral (Fig. 3). Con estos análisis se insiste en el interrogatorio, que revela un 


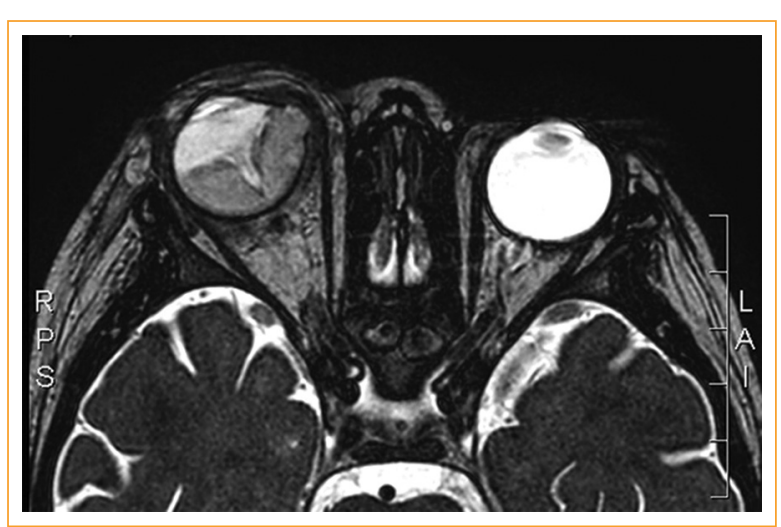

Figura 3. Corte axial de resonancia magnética en T2 en la que se objetivan el compromiso inflamatorio difuso de partes blandas en la órbita derecha y la presencia de un estafiloma anterior medial, asociado a la inserción del recto medio, compatible con solución de continuidad escleral.

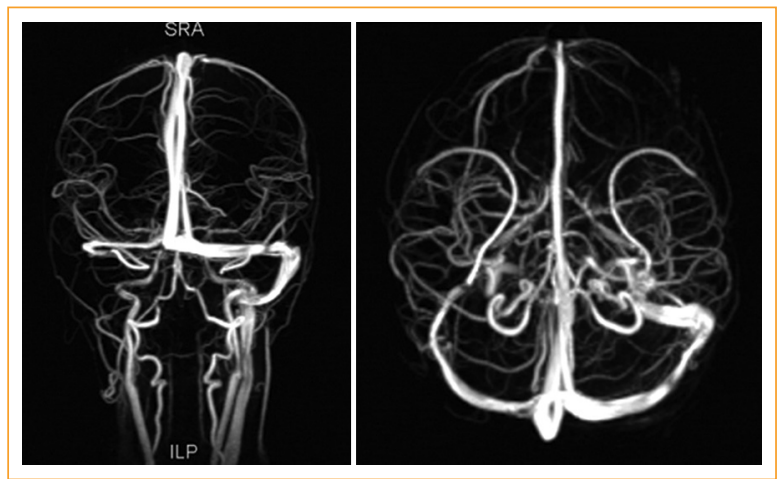

Figura 4. Angiorresonancia que muestra un defecto del lleno central de los senos sigmoideo y transverso derechos, compatible con trombosis.

trauma de origen inespecífico. El paciente recibe tratamiento antibiótico intravenoso con vancomicina $(60 \mathrm{mg} /$ $\mathrm{kg}$ al día) y ceftazidima (150 mg/kg al día), y progresa con buena evolución clínica.

El servicio de diagnóstico por imágenes detectó en la angiorresonancia un defecto del lleno central de los senos sigmoideo y transverso derechos, compatible con trombosis (Fig. 4). Se indican tratamiento anticoagulante con heparina, hemocultivos y cirugía exploratoria con toma de muestra. Los hemocultivos fueron negativos. En la exploración quirúrgica, una semana luego de la internación y compensado el cuadro sistémico, se observó daño del recto medio y de la esclera, se realiza toma de muestra para cultivos con resultados negativos.
Se interpreta el cuadro como endoftalmitis y celulitis orbitaria de origen traumático con trombosis de senos transverso y sigmoideo derechos, sin compromiso del seno cavernoso homolateral. El paciente tiene el alta hospitalaria luego de 21 días de internación con antibióticos sistémicos por vena y anticoagulantes. Se evitaron la ptisis ocular y las complicaciones graves, como sepsis, meningitis e incluso la muerte. Seis meses después del diagnóstico se discontinuó el tratamiento anticoagulante con enoxaparina por buena evolución.

\section{Discusión}

La trombosis de senos transverso y sigmoideo es un cuadro clínico infrecuente, pero que puede ser mortal. El diagnóstico temprano muchas veces es una tarea difícil porque los pacientes no suelen presentar al inicio del cuadro síntomas específicos, solo quizás los correspondientes al punto de partida de la infección. Aún puede resultar de mayor dificultad en casos pediátricos, ya que muchas veces la anamnesis no puede aportar los datos necesarios ${ }^{1-3,6}$.

Desde el comienzo de la era de los antibióticos la mortalidad en estos pacientes ha descendido drásticamente, desde casi el $100 \%$ a menos del $15-20 \%$. He aquí donde radica la importancia de su conocimiento para lograr indicar el tratamiento que requieren estos pacientes. Para esto es necesario comprender algunos aspectos anatómicos. El seno transversal conecta la tórcula de Herófilo al seno sigmoideo. Recibe una serie de importantes venas supratentoriales procedentes de los lóbulos occipital y temporal, especialmente la vena anastomótica inferior, también denominada vena de Labbé. Muchas venas infratentoriales drenan en este seno, así como en el seno petroso superior, que recibe canales venosos del compartimento supratentorial e infratentorial y de las estructuras del tímpano. El seno sigmoideo es la continuación inferior del seno transverso 5,7 .

Para el estudio de este complejo entramado es necesario valerse de pruebas complementarias como la angiorresonancia. El uso de la angiorresonancia con gadolinio como medio de contraste revela una característica distintiva en la trombosis sinusal transverso-sigmoidea. La trombosis se manifiesta como una señal de tejido blando rodeada por las paredes del seno dural brillantemente reforzadas por el medio de contraste, debido a que son vasculares. Por lo general, la configuración es triangular. 
Una apariencia similar se observa en el seno transversal. Esta imagen triangular se conoce como "signo delta» y se ve también en la tomografía computada ${ }^{2,6}$.

La puerta de entrada de la infección suele ser en el macizo facial. Las celulitis aisladas o junto con endoftalmitis rara vez producen entre sus complicaciones trombosis sépticas de senos venosos craneales, y no hemos encontrado reportes similares al caso presentado. Por lo general existe asocición a trombosis séptica de seno cavernoso, que también es un cuadro clínico de extrema gravedad por sus relaciones anatómicas. El diagnóstico clínico suele establecerse de manera más temprana que en otros senos venosos por la temprana afectación de ciertos nervios craneales (III, IV, V1, V2 y VI) que transcurren por él.

\section{Conclusiones}

La frecuencia de trombosis de senos transverso y sigmoideo es baja, y la edad y el sexo no tienen relación con su presentación. En casos como este, la angiorresonancia sirve como prueba complementaria para que el diagnóstico se realice oportunamente y poder indicar el tratamiento necesario para salvar la vida del paciente.

\section{Financiamiento}

Los autores no recibieron ningún financiamiento para la realización de este artículo.

\section{Conflicto de intereses}

Todos los autores declaran que no existen conflictos de intereses.

\section{Responsabilidades éticas}

Protección de personas y animales. Los autores declaran que para esta investigación no se han realizado experimentos en seres humanos ni en animales.

Confidencialidad de los datos. Los autores declaran que han seguido los protocolos de su centro de trabajo sobre la publicación de datos de pacientes.

Derecho a la privacidad y consentimiento informado. Los autores han obtenido el consentimiento informado de los pacientes y/o sujetos referidos en el artículo. Este documento obra en poder del autor de correspondencia.

\section{Bibliografía}

1. Chiewvit P, Piyapittayanan $S$, Poungvarin N. Cerebral venous thrombosis: diagnosis dilemma. Neurol Int. 2011;3:13.

2. Weon YC, Marsot-Dupuch K, Ducreux D, Lasjaunias P. Septic thrombosis of the transverse and sigmoid sinuses: imaging findings. Neuroradiology. 2005;47:197-203.

3. Moscote-Salazar L, Alcala-Cerra G, Alvis-Miranda H, Castellar-Leones S. Cerebral sinus venous thrombosis. J Neurosci Rural Pract. 2013;4:427.

4. Teichgraeber JF, Per-Lee JH, Turner JS. Lateral sinus thrombosis: a modern-day perspective. Laryngoscope. 1982;92:744-51.

5. McArdle CB, Mirfakhraee M, Amparo EG, Kulkarni MV. MR imaging of transverse/sigmoid dural sinus and jugular vein thrombosis. J Comput Assist Tomogr. 1987;11:831-8.

6. Sebire G. Cerebral venous sinus thrombosis in children: risk factors, presentation, diagnosis and outcome. Brain. 2005;128:477-89.

7. Massrey C, Altafulla JJ, Iwanaga J, Litvack Z, Ishak B, Oskouian RJ, et al. Variations of the transverse sinus: review with an unusual case report. Cureus. 2018;10:e3248. 